\title{
Research on Adaptive Equilibrium Scheduling Model of Big Data Based on Internet of Things Transmission
}

\author{
Fang Meng ${ }^{1, a}$, Guogen Fan ${ }^{2, b}$ \\ ${ }^{1}$ Huali College Guangdong University of Technology, Guangdong, Guangzhou 511325, China. \\ ${ }^{2}$ Guangzhou Huali Science and Technology Vocational College, Guangzhou 511325, China. \\ ayuhu0422@163.com, b29942978@qq.com
}

Keywords: Big Data, Adaptive Equilibrium Scheduling Model

\begin{abstract}
The big data transmitted by the Internet of Things is affected by the inter-code interference of the transmission channel, which will cause large scheduling delays and bit errors. In this paper, a big data adaptive equilibrium scheduling model based on decision feedback equalization was proposed to improve the big data scheduling and adaptive equalization control capability of the Internet of Things transmission. In order to improve the big data scheduling and adaptive equalization control capability of the Internet of Things transmission. The transverse filtering control algorithm was used to optimize the performance of IoT transmission, and the IoT transmission channel model was constructed. The inter-code interference filtering method was adopted, and the big data scheduling anti-interference design of the Internet of Things transmission was executed. Based on bandwidth modulation and baud interval equalization control technology, large data transmission and adaptive tuning of the Internet of Things transmission communication system were performed; The channel equalization control model for the Internet of Things to transmit big data was constructed, and the maximum likelihood estimation value of the big data adaptive equalization scheduling was calculated; The IoT transmission big data fuzzy clustering process was implemented to realize the big data adaptive equalization scheduling of the Internet of Things transmission network. According to the research, based on the method in this paper, the balance of the big data scheduling of the Internet of Things was better, the anti-interference ability was stronger, and the output bit error rate was lower. The method in this paper has good application value in the design of IoT transmission and communication optimization.
\end{abstract}

\section{Introduction}

With the development of Internet of Things (IoT) communication technology, the adoption of the Internet of Things for large data transmission has become the main direction of future wireless data transmission and communication applications. In the process of big data transmission, the Internet of Things needs to dynamically allocate IP addresses and bandwidths, which results in poor channel equalization, steady-state scheduling of data output, and high bit error rate. In order to improve the stable scheduling capability of IoT communication data and the quality of IoT transmission, it is necessary to perform balanced scheduling of large data transmission in the Internet of Things. The big data adaptive equilibrium scheduling model for researching IoT transmission is of great significance in IoT communication ${ }^{[1]}$. The construction of the big data adaptive equilibrium scheduling model for IoT transmission is based on the statistical analysis and feature extraction of big data bit information flow. The large-data transmission mediums transmitted by the Internet of Things have large channel sizes, large types, and are characterized by time-varying and random changes. Because the data association attribute changes progressively to the cluster clustering center, the conventional big data scheduling method cannot effectively and accurately mine the target data, resulting in distortion of the channel output ${ }^{[2]}$.

In the traditional method, the big data equalization scheduling method for the Internet of Things transmission of the Internet of Things mainly includes a baud interval equalization scheduler, a spectrum analysis scheduling method, and a fractional interval equalization scheduling method ${ }^{[3]}$. 
Combined with sampling statistical analysis, big data scheduling is performed to improve the channel equalization of Internet of Things transmission ${ }^{[4]}$. In reference ${ }^{[5]}$, an IoT transmission big data adaptive equalization scheduling algorithm based on association feature fusion is proposed: high-dimensional phase space reconstruction of IoT transmission big data distributed information flow is implemented; In the phase space, the correlation feature quantity of the big data transmitted by the Internet of Things is extracted to realize channel equalization control and big data scheduling. However, the method has large computational overhead and low real-time performance for the IoT transmission big data scheduling. In reference ${ }^{[6]}$, a large data clustering equilibrium scheduling method for IoT transmission based on particle swarm differential perturbation optimization is proposed. Based on the data mining and clustering analysis methods, the Internet of Things transmission data containing interference components is accurately extracted from the storage medium. However, with this method, the fusion of adaptive equalization scheduling of the Internet of Things with large correlation data is not good.

Aiming at the above problems, in this paper, a big data adaptive equilibrium scheduling model based on decision feedback equalization was proposed. First, based on the lateral filtering control algorithm, the optimized design of the IoT transmission performance was implemented, and the IoT transport channel model was constructed; Then, the Internet of Things transmission big data fuzzy clustering process was executed to implement big data adaptive equalization scheduling for the Internet of Things transmission network; Finally, the simulation experiment was carried out. The results showed that the proposed method had superior performance in improving the big data adaptive equalization scheduling capability of IoT transmission.

\section{IoT transport channel model and data feature analysis}

\subsection{Analysis of the Internet of Things Transmission Channel Model}

In order to achieve accurate and balanced scheduling of big data transmission in the Internet of Things, in the IoT communication system, the reorganization of big data distributed information and the construction of the channel model are implemented. According to the channel reconstruction analysis of the big data transmission of the Internet of Things, the transmission scheduling is carried out ${ }^{[7]}$.

There are the following assumptions: the sample category for the transmission of big data in the Internet of Things is $\omega_{i}$,the span of the IoT transmission big data scheduling channel is $p$,data stream sample is $S=\overline{X_{1}}, \overline{X_{2}}, \cdots, \overline{X_{k}}, \cdots$. The attribute set of the IoT transmission big data distribution sample collected in the two sets of channels is $i \in S_{s}$ the corresponding equivalent channel impulse response function satisfies the following formula:

$$
\alpha^{T} Q \alpha=\sum_{i=1}^{n} \sum_{j=1}^{n} \alpha_{i} \alpha_{j} Q_{i j} \geq 0
$$

The data set to be balanced scheduled contains $\mathrm{n}$ samples; where, the bit sequence transport stream of the, sample ${ }^{x_{i}},{ }^{i=1,2, \cdots, n}$ in the IoT communication channel can be expressed as:

$$
s(t)=\sum_{m=-\infty}^{\infty} \sum_{n=-\infty}^{\infty} a_{m n} g_{m n}(t)+n(t)
$$

where, ${ }^{a_{m n}}$ represents the envelope magnitude of potentially useful information for big data transmitted by the Internet of Things to be evenly scheduled. ${ }^{g_{m n}}(t)$ represents the statistical average of the data, ${ }^{n(t)}$ represents the interference term.

Assume that the big data transmission system consists of $N=2 P$ IoT transmission communication system points. In the multipath transmission of the IoT transmission communication system, the shortest distance of each data receiving node is defeatured analysis of the random fading channel for big data transmission is performed ${ }^{[8]}$. IoT transmission communication system of the 
big data transmission channel is an extended channel, and the multipath channel model of big data transmission is expressed as:

$$
x_{m}(t)=\sum_{i=1}^{I} s_{i}(t) e^{j \varphi_{m i}}+n_{m}(t),-p+1 \leq m \leq p
$$

where, ${ }^{s_{i}}(t)$ represents the received data feature quantity of the i node in the IoT transmission communication node, ${ }^{x_{m}}(t)$ represents the bit rate of big data information received by the Internet of Things transmission communication. Under the finite transmission vector set, the IoT transmission channel has multipath characteristics, and the IoT transmission channel model is constructed, as shown in Figure 1.

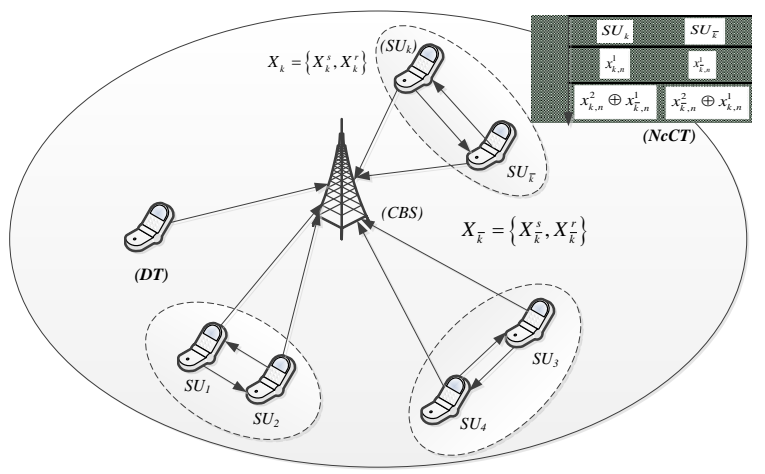

Figure 1. Channel model of IoT data transmission

According to the channel model shown in Fig. 1, the inter-symbol interference filtering method is adopted for the big data scheduling anti-interference design of the Internet of Things transmission; the bandwidth modulation and the baud interval equalization method are used for the equalization design of the big data scheduling ${ }^{[9]}$.

\subsection{Feature extraction and quantitative analysis of big data transmission}

In the big data transmission channel of the Internet of Things communication network, the relationship between channel attenuation and channel spreading in a single frame data transmission time is expressed as:

$$
K_{v}(z)=1-\prod_{i=1}^{N}\left[\left(1-P_{d i}\right)\left(1-P_{e i}\right)+P_{d i} P_{e i}\right]
$$

The IEEE802.3EFM communication protocol is used to construct the Internet of Things transmission protocol. The linear equalization technology is used to adaptively synthesize large data in the Internet of Things communication network, and the multipath characteristics of the big data transmission channel of the Internet of Things communication network are analyzed. An adaptive iterative function is constructed, and the feature quantization analysis of big data scheduling is performed by using bandwidth modulation, and an iterative function is obtained, which is expressed as:

$$
d(t)=a(t) c(t)=\sum_{n=0}^{\infty} d_{n} g_{c}\left(t-n T_{c}\right)
$$

where:

$$
d_{n}=\left\{\begin{array}{ll}
+1 & a_{n}=c_{n} \\
-1 & a_{n} \neq c_{n}
\end{array} \quad(n-1) T_{c} \leq t \leq n T_{c}\right.
$$

Taking the extracted feature quantity as the test set, the adaptive learning training of the Internet of Things to transmit big data is executed, and the adaptive training weight of the Internet of Things to transmit big data is obtained, which is expressed as $\omega=\left(\left(\omega_{1}, a_{1}^{\prime}\right),\left(\omega_{2}, a_{2}^{\prime}\right), \ldots,\left(\omega_{\mathrm{n}}, a_{\mathrm{n}}^{\prime}\right)\right)^{T}, \omega_{\mathrm{j}} \in[0,1]$. 
The offset load in the transport channel is calculated and expressed as:

$$
x_{i}(n)=\sum_{j=1}^{M} h_{i j}(n)^{T} \boldsymbol{s}_{j}(n)+v_{i}(n)
$$

Using the inter-symbol interference filtering method, the big data scheduling anti-interference design of the Internet of Things transmission is implemented, and the filter transfer function is designed, which is expressed as:

$$
y_{j}(n)=\sum_{i=1}^{P} f_{i j}(n)^{T} \boldsymbol{x}_{i}(n)
$$

Where, $f_{i j}$ represents the load frequency of the two groups of IoT transmission big data.

Considering the transmission channels of two different attributes, the load balancing control is implemented. The weighting factor for load balancing is $W_{c}<\delta\left(t_{c}, t_{a}\right)$, and $\delta\left(t_{c}, t_{a}\right)=\frac{2^{-\lambda\left(t_{c}-t_{a}+T_{d}\right)-1}}{2^{-\lambda T_{d}}-1}$, the symbol of big data transmission in the Internet of Things is $A[n+1]$, and $A[j]=A[j+1]$.

Based on the random phase spread spectrum method, symbol sequence modulation is performed to realize feature extraction and quantization analysis of the Internet of Things transmission big data.

\section{Optimization of big data equilibrium scheduling model}

\subsection{Channel equalization design}

In this part, the balanced scheduling design of big data for the Internet of Things is carried out. In this paper, a big data adaptive equilibrium scheduling model based on decision feedback equalization is proposed ${ }^{[10]}$.

The statistical average of big data transmissions in the Internet of Things is $\sum_{-\infty}^{\infty} \cdots \sum_{-\infty}^{\infty}\left|c_{m x}\left(\tau_{1}, \cdots, \tau_{m-1}\right)\right|<\infty, m=1,2, \cdots, 2 k$ , with $\mathrm{L}$ equalizers, the equalization design of the channel is performed. The result is shown in Figure 2.

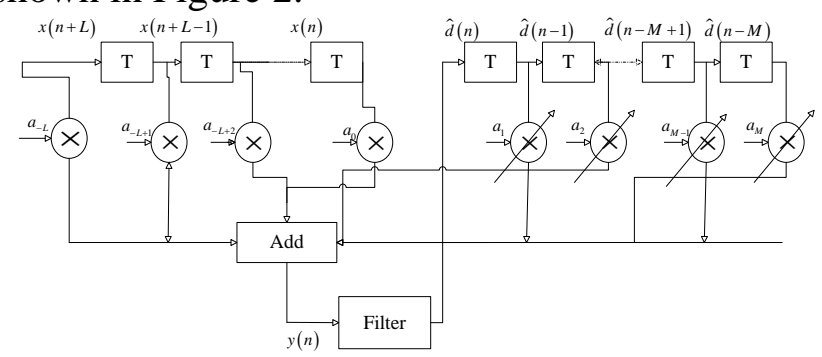

Figure 2. The channel equalizer design of the IoT big data transmission

According to the equalizer designed in Fig. 2, channel equalization control is performed. In the $\mathrm{k}$ time slice, the Internet of Things transmission big data is gathered into the big data dispatch center of the Internet of Things transmission network. $V=\left[v_{1}, v_{2}, \ldots, v_{n}\right]$ represents a big data scheduling vector.

For the IoT transmission network, the maximum quantization resource aggregation flow of the adaptive equalization scheduling set of big data has the following conditions: $\hat{q}_{i+1, i}:=c_{i+1, i}$ and $\hat{q}_{i^{\prime}, i}:=d_{i^{\prime}, i}$. When $c_{i, i-1} \leq \min \left\{c_{i+1, i}, d_{i^{\prime}, i}\right\}$,the large adaptive forwarding feature quantity of the Internet of Things is expressed as:

$$
s_{m}(t)=\cos \left\{2 \pi f_{0}\left[t+\tau_{m}(\theta)\right]\right\}
$$

The covariance of the associated features of the Internet of Things transmission big data is 
extracted; with frequency domain equalization control, the load balancing scheduling is performed. The process of frequency domain equalization is described as:

$$
u_{(i, d)}= \begin{cases}z_{(i, d)} & \text { rand } \leq C_{R}, d=r n_{i} \\ x_{(i, d)} & \text { other }\end{cases}
$$

Where $\left\{x_{(i, d)}, z_{(i, d)}, u_{(i, d)}\right\}$ represents the channel compensation coefficients of the Internet of Things communication network, ${ }^{C_{R}}$ represents the balance control coefficient of IoT big data, ${ }^{r n_{i}}$ represents the random integer. Based on the baud interval sampling analysis method, the load distribution of the big data transmitted by the Internet of Things is obtained, which is expressed as:

$$
\left\{\begin{array}{l}
v_{i d}^{(t+1)}=w * v_{i d}^{t}+c_{1} * r_{1}\left(p_{i d}^{t}-x_{i d}^{t}\right)+c_{2} * r_{2}\left(p_{g d}^{t}-x_{i d}^{t}\right) \\
x_{i d}^{(t+1)}=x_{i d}^{t}+v_{i d}^{(t+1)}
\end{array}\right.
$$

Combined with the adaptive forwarding control strategy of the transmission link, the clustering processing of the big data transmission of the Internet of Things is carried out based on the joint rule constraint method, and finally the equilibrium scheduling control is performed according to the clustering fusion result.

\subsection{Channel spread spectrum and equalization scheduling output}

The channel equalization control model for the transmission of big data in the Internet of Things is constructed; the maximum likelihood estimation value of the big data adaptive equalization scheduling is calculated, which is expressed as:

$$
\mathrm{z}_{(i, d)}=x_{r 1}+F *\left(x_{r 2}-x_{r 3}\right)
$$

Where $F$ represents the spreading factor. The spread spectrum sequence corresponding to the big data adaptive equalization scheduling node of the Internet of Things transmission network is defined as:

$$
u_{(i, d)}^{k+1}= \begin{cases}x_{i d}^{(t+1)} & f_{\text {fitness }}^{t}<f_{\text {fitness }}^{*} \\ z_{(i, d)}^{(k+1)} & f_{\text {fitness }}^{t} \geq f_{\text {fitness }}^{*}\end{cases}
$$

Where, the candidate solution for the big data spread spectrum processing of the Internet of Things transmission is $u_{i}^{(k+1)}=\left\{u_{i 1}^{(k+1)}, u_{i 2}^{(k+1)}, \ldots, u_{i D}^{(k+1)}\right\}$ and the optimal solution is $x_{i}^{k}=\left\{x_{i 1}^{k}, x_{i 2}^{k}, \ldots, x_{i D}^{k}\right\}$. $x_{r 1}$ is set to the base vector and $\left\{x_{r 2}, x_{r 3}\right\}$ is set to the difference vector, and fuzzy clustering processing of the Internet of Things to transmit big data is performed to implement adaptive equalization scheduling of big data of the Internet of Things transmission network.

\section{Simulation experiment analysis}

In order to test the application performance of the proposed method in the big data adaptive equalization scheduling of the Internet of Things transmission network, the simulation experiment was carried out. The experiment was designed using Matlab7. The bandwidth of big data sampling in the Internet of Things transmission network is 10Gbps. The internet of things transmission big data scale set is 10,000 . The test sample set is 200 . The disturbance error coefficient of the Internet of Things data transmission is set to $\beta=0.001$. The adjustment factor for the channel equalization configuration is 0.12 . The initial sampling interval for the transmission of big data in the Internet of things is $t_{1}=10 \mathrm{~ms}$. The termination frequency is $f_{2}=2.12 \mathrm{~Hz}$. According to the above simulation 
parameter setting, the IoT transmission big data scheduling analysis is performed, and the input IoT transmission big data sample is obtained as shown in Fig. 3.

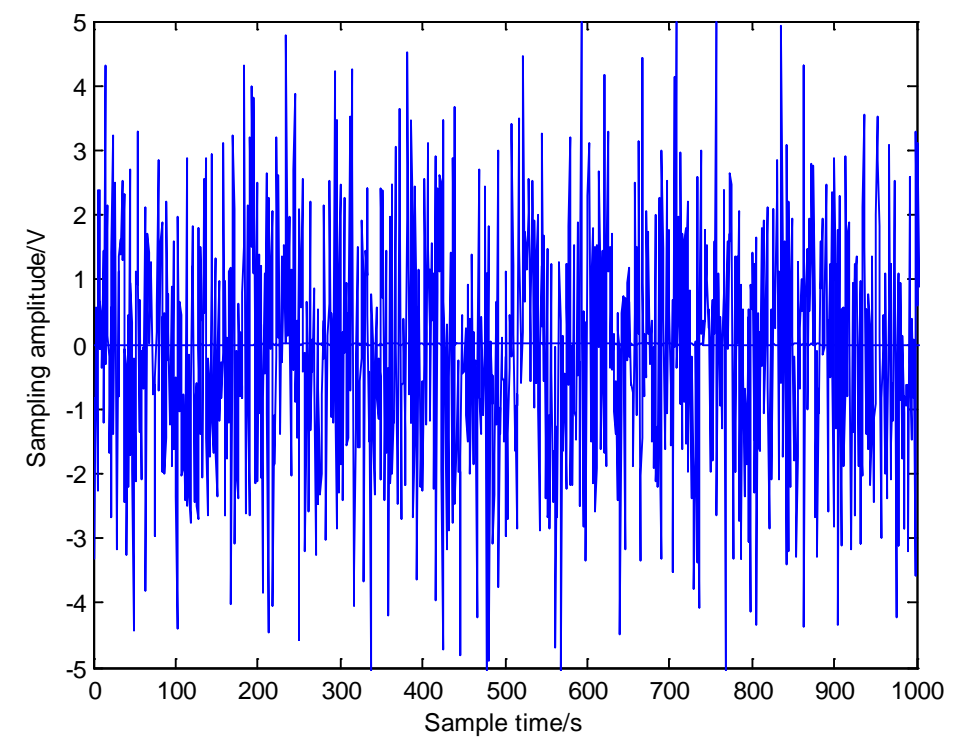

Figure 3.Input IoT transmission big data sample

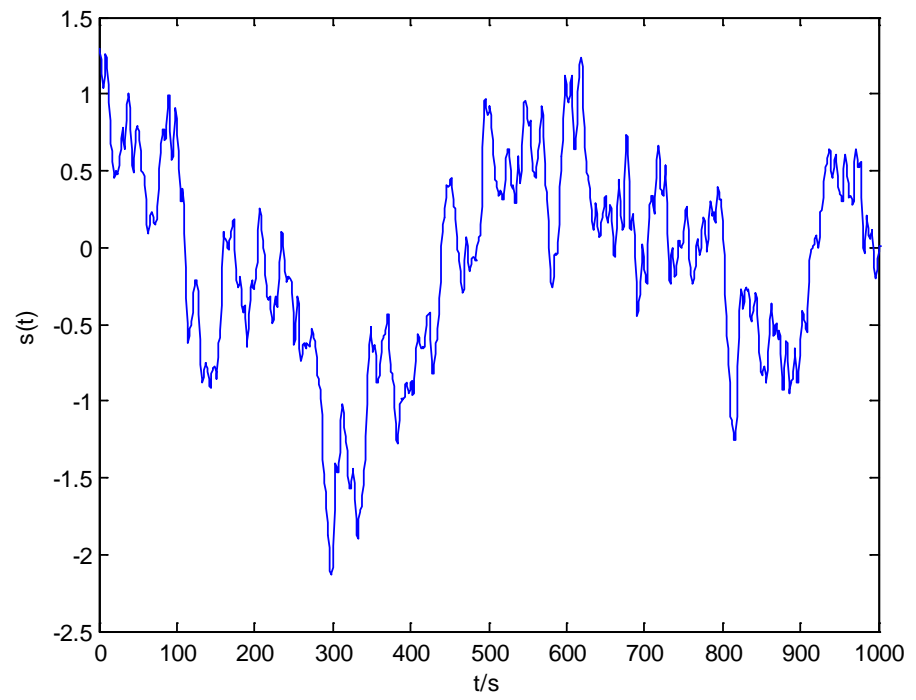

Figure 4.Equilibrium scheduling output IoT transmission big data

Interference suppression processing is performed on the Internet of Things transmission big data collected in Figure 3. Large-data scheduling anti-jamming design for IoT transmission using intersymbol interference filtering. Bandwidth modulation and baud interval equalization control technology are used for large data transmission and adaptive scheduling of IoT transmission communication systems. The big data that gets the balanced scheduling output is shown in Fig. 4.

Based on the analysis of the balance and rationality of the big data scheduling results, the separation performance of the noisy data signal model is analyzed, and the interference noise and noiseless signal component output are obtained as shown in Fig. 5. 


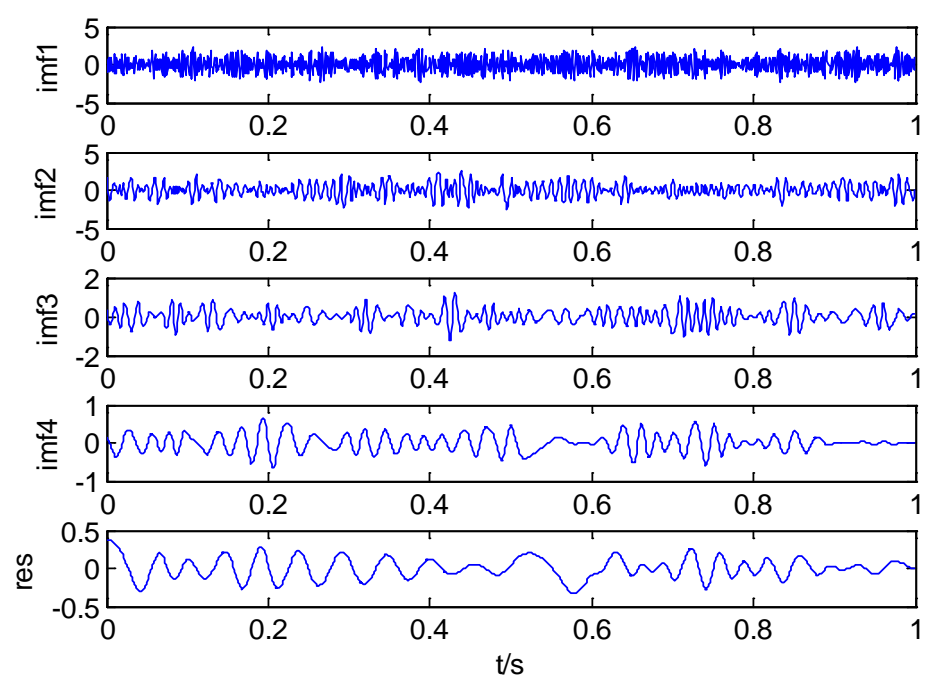

Figure 5.Big data scheduling output

Analysis of Figure 5 shows that the denoising ability of IOT for large data scheduling is better. Load balancing control is strong. The output bit error rate is used as the test index, and the output performance of different methods for big data scheduling is analyzed. The comparison results are shown in Table 1. Analysis of the results of Table 1 shows that the proposed method performs the big data scheduling of the Internet of Things transmission and can control the output bit error rate to 0 when the interference signal-to-noise ratio is $-6 \mathrm{~dB}$. The average output error is reduced and the quality of IoT transmission is improved.

Table 1. Output performance test

\begin{tabular}{cccc}
\hline input signal to noise ratio /dB & the method in this paper & reference [4] & reference [6] \\
\hline-10 & 0.025 & 0.201 & 0.087 \\
-8 & 0.012 & 0.065 & 0.075 \\
-6 & 0 & 0.064 & 0.049 \\
-4 & 0 & 0.022 & 0.025 \\
\hline
\end{tabular}

\section{Conclusion}

In this paper, a big data adaptive equilibrium scheduling model based on decision feedback equalization was proposed. The transverse filtering control algorithm was used to optimize the performance of IoT transmission, and the IoT transmission channel model was constructed. The inter-code interference filtering method was adopted, and the big data scheduling anti-interference design of the Internet of Things transmission was executed. Based on bandwidth modulation and baud interval equalization control technology, large data transmission and adaptive tuning of the Internet of Things transmission communication system were performed; The channel equalization control model for the Internet of Things to transmit big data was constructed, and the maximum likelihood estimation value of the big data adaptive equalization scheduling was calculated; The IoT transmission big data fuzzy clustering process was implemented to realize the big data adaptive equalization scheduling of the Internet of Things transmission network. According to the research, based on the method in this paper, the balance of the big data scheduling of the Internet of Things was better, the anti-interference ability was stronger, and the output bit error rate was lower. The method in this paper has good application value in the design of IoT transmission and communication optimization.

\section{References}

[1] XIAO Wen, HU Juan. Performance analysis of frequent itemset mining algorithms based on 
sparseness of dataset[J]. Journal of Computer Applications, 2018, 38(4): 995-1000.

[2] LI Zhixiang, LI Yun, HE Liang. Decomposition multiobjective optimization algorithm with new neighborhood model[J]. Computer Engineering and Application, 2018, 54(14): 1-6.

[3] GUO H P, DONG Y D, MAO H T, et al. Logistic discrimination based rare-class classification method[J]. Journal of Chinese Computer Systems, 2016, 37(1):140-145.

[4] YANG Yuxiang, XIA Changxiong, and TONG Wuqin. Estimation of the time varying TDOA and FDOA in GEO-LEO dual-satellites location system[J]. Signal Processing, 2012, 28(10): 14651474.

[5] HAO S G, ZHANG L, and MUHAMMAD G. A union authentication protocol of cross-domain based on bilinear pairing[J]. Journal of Software, 2013, 8(5): 1094-1100.

[6] DONG G L, RYU K S, BASHIR M, et al. Discovering medical knowledge using association rule mining in young adults with acute myocardial infarction[J]. Journal of Medical Systems, 2013, 37(2):1-10.

[7] KHALILI A, SAMI A. SysDetect:a systematic approach to critical state determination for industrial intrusion detection systems using Apriori algorithm[J]. Journal of Process Control, 2015, 2776:154-160.

[8] KESHAVAMURTHY B N, KHAN A M, TOSHNIWAL D. Privacy preserving association rule mining over distributed databases using genetic algorithm[J]. Neural Computing \& Applications, 2013, 22(Supplement 1):351-364.

[9] BI Anqi, WANG Shitong. Transfer Affinity Propagation Clustering Algorithm Based on Kullback-Leiber Distance [J]. JOURNAL OF ELECTRONICS AND INFORMATION, 2016, 38(8): 2076-2084.

[10] LONG M, WANG J, DING G, et al. Adaptation regularization: A general framework for transfer learning [J]. IEEE Transactions on Knowledge and Data Engineering, 2014, 26(5): 10761089. 